\title{
Chirality-Dependent Electron Spin Filtering by Molecular Monolayers of Helicenes
}

\author{
Matthias Kettner, ${ }^{\dagger}$ Volodymyr V. Maslyuk, ${ }^{\ddagger}$ Daniel Nürenberg, ${ }^{\dagger}$ Johannes Seibel, $^{\S}$ Rafael Gutierrez, ${ }^{\ddagger}$

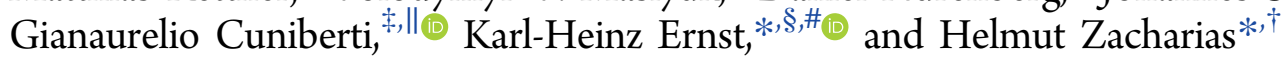 \\ ${ }^{\dagger}$ Center for Soft Nanoscience and Physikalisches Institut, University of Münster, 48149 Münster, Germany \\ ${ }^{\ddagger}$ Institute for Materials Science and Max Bergmann Center of Biomaterials, TU Dresden, 01062 Dresden, Germany \\ ${ }^{\S}$ Empa, Swiss Federal Laboratories for Materials Science and Technology, 8600 Dübendorf, Switzerland \\ "Dresden Center for Computational Materials Science and Center for Advancing Electronics Dresden, TU Dresden, 01062 Dresden, \\ Germany \\ \#Department of Chemistry, University of Zurich, 8057 Zürich, Switzerland
}

Supporting Information

\begin{abstract}
The interaction of low-energy photoelectrons with well-ordered monolayers of enantiopure helical heptahelicene molecules adsorbed on metal surfaces leads to a preferential transmission of one longitudinally polarized spin component, which is strongly coupled to the helical sense of the molecules. Heptahelicene, composed of only carbon and hydrogen atoms, exhibits only a single helical turn but shows excess in longitudinal spin polarization of about $P_{Z}=6$ to $8 \%$ after transmission of initially balanced left- and right-handed spin polarized electrons. Insight into the electronic structure, that is, the projected density of states, and the spin-dependent electron scattering in the helicene molecule is gained by using spin-resolved density functional theory calculations and a model Hamiltonian approach, respectively. Our results support the semiclassical picture of electronic transport along a helical pathway under the influence of spin-orbit coupling induced by the electrostatic molecular potential.
\end{abstract}

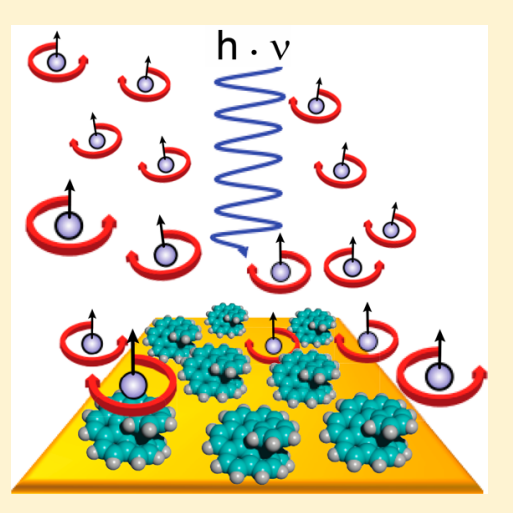

hirality-the occurrence of objects being either left- or right-handed-plays a pre-eminent role in the biological world as well as in chemical and materials science. ${ }^{1}$ This includes enantioselective catalysis of chiral molecules, ${ }^{2}$ liquid crystal displays, ${ }^{3}$ and dissociative electron attachment. ${ }^{4}$ Early work of Farago and Kessler showed that spin-polarized electrons scatter asymmetrically from chiral molecules when they contain a heavy metal atom; ${ }^{5,6}$ however, the magnitude of the differential effect was only on the order of $10^{-4}$. Recently, it has been shown that surface-oriented helical polymers, such as DNA or oligopeptides, can show a substantial filtering effect for spin-polarized electrons, with a strong preference for the transmission of only one spin component. ${ }^{7,8}$ It is anticipated that this property is expected to be highly beneficial for applications in spintronic devices or for enantioselective chemistry, ${ }^{9,10}$ in particular, for spin-polarized electron sources on the nanoscale. However, the physical origin of this effect, also termed "chirality-induced spin selectivity (CISS)", ",11 has not yet been fully elucidated and is still the subject of intensive debate. $^{12-17}$

Because of their outstanding optical properties, conjugated organic helical molecules, so-called helicenes, are promising candidates for new functional materials and devices. ${ }^{18,19} \mathrm{~A}$ recent model calculation predicts energy-dependent longitudinal spin polarizations, that is, polarized parallel to the surface normal, of up to $P_{Z}=25 \%$ for electrons transmitting through all-carbon helicenes with three full helical turns. ${ }^{20}$ The molecule heptahelicene $\left([7] \mathrm{H}, \mathrm{C}_{30} \mathrm{H}_{18}\right.$, Figure 1a) comprises seven [a,c]annulated benzo groups, which introduce steric overcrowding such that not all rings fit into a single plane, thus leading to a single helical turn. ${ }^{21}$ Consequently, two mirror-related enantiomers exist, $(M)$ - and $(P)$-heptahelicene ( $M$ for minus, $P$ for plus). The adsorption of $[7] \mathrm{H}$ has been previously studied on various single-crystal metal surfaces, ${ }^{22}$ where its lower rings are basically aligned parallel to the surface and the rest spiral upward (Figure 1b). ${ }^{23}$ For the pure enantiomers, defined monolayer structures have been observed on $\mathrm{Cu}(332)$, $\mathrm{Cu}(111), \mathrm{Cu}(100), \mathrm{Ag}(100), \mathrm{Ag}(111)$, and $\mathrm{Au}(111) .^{23-27}$ The corresponding example for $\mathrm{Cu}(111)$ is shown in Figure 1c.

To shine light into the mechanism of CISS, we present a study performed with monolayers of heptahelicene molecules on copper, silver, and gold surfaces. Photoelectrons for transmission through the molecular layer are generated in the metallic substrate by ultraviolet laser pulses $(\lambda=213 \mathrm{~nm}$; $h \nu=$ $5.83 \mathrm{eV}$ ) (Figure 1d). Already a monomolecular enantiopure layer shows a pronounced electron spin filtering effect, as identified with a Mott polarimeter (Figure 1d). Monolayers

Received: January 22, 2018

Accepted: April 4, 2018

Published: April 4, 2018 
a
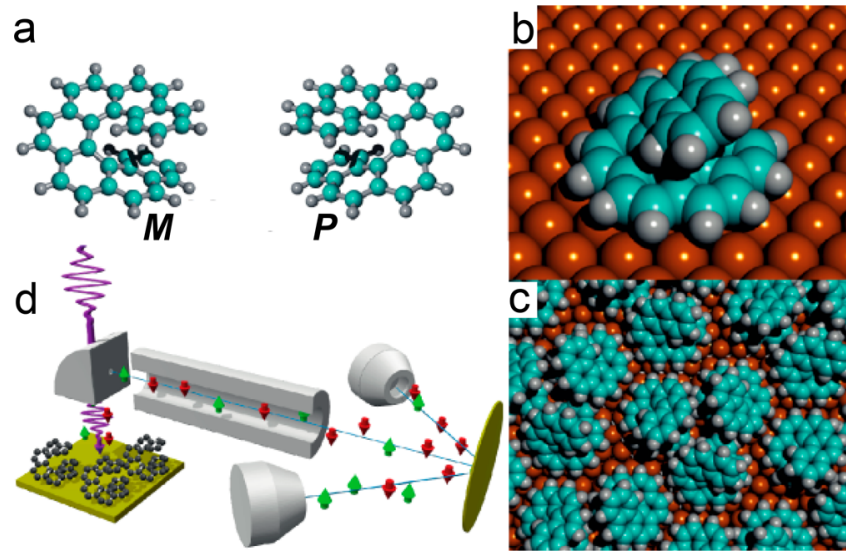

Figure 1. (a) Ball-and-stick models of the two enantiomers of [7] H. $(M)-[7] \mathrm{H}$ marks the left-handed helical sense and $(P)-[7] \mathrm{H}$ the righthanded sense. (b) Sketch of orientation of a single $(M)-[7] \mathrm{H}$ on a metal surface. (c) Sketch of monolayer structure of $(M)-[7] \mathrm{H}$, as observed on $\mathrm{Cu}(111)$. (d) Experimental setup of the photoemission experiment including the Mott analyzer (see the text for details).

with molecules of opposite helical sense cause inversion of the preferred spin direction, delivering unprecedented insight into the relation between helical sense and the sign of preferred electron spin polarization. The experimental studies are complemented by first-principles calculations of the electronic structure of helicene on $\mathrm{Cu}(332), \mathrm{Ag}(110)$, and $\mathrm{Au}(111)$ and by a phenomenological Hamiltonian model approach for the evaluation of the transmitted spin polarization.

Figure 2 shows histograms of measured longitudinal spin polarization $P_{Z}$ of electrons transmitted through $[7] \mathrm{H}$ on $\mathrm{Cu}(332) . \mathrm{P}_{Z}$ is defined as:

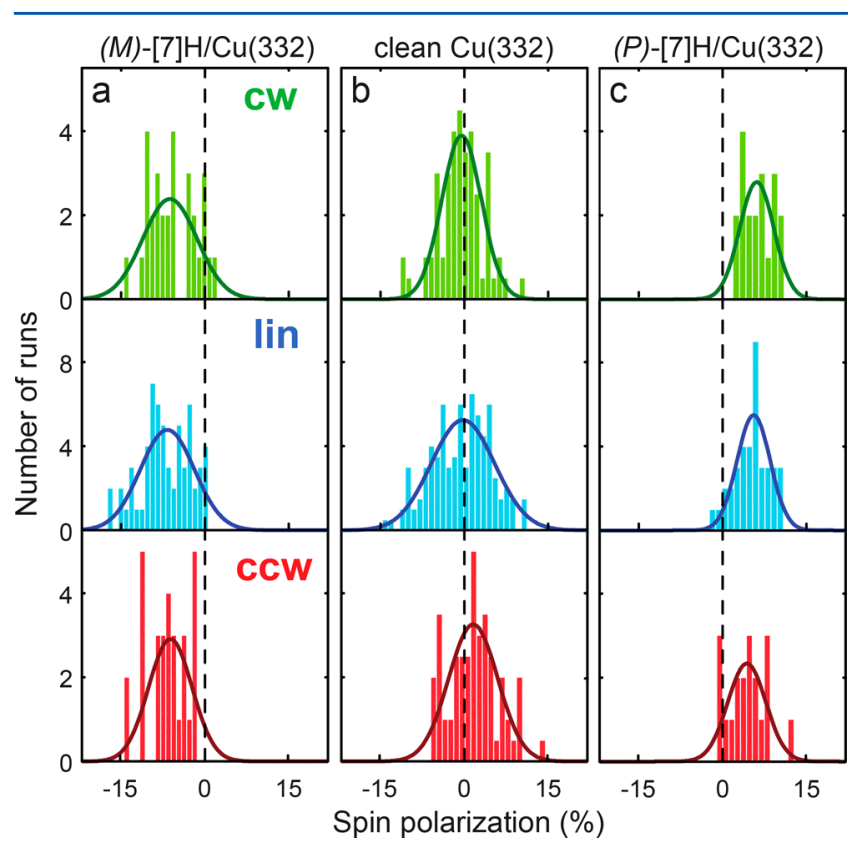

Figure 2. Spin polarization of electrons from (a) $(M)-[7] \mathrm{H},(\mathrm{b})$ clean $\mathrm{Cu}(332)$, and $(\mathrm{c})(P)-[7] \mathrm{H}$ on $\mathrm{Cu}(332)$. Upper (green) and lower (red) rows mark experiments performed with clockwise circular and counterclockwise circular polarized UV light, respectively. The middle row (blue) shows experiments performed with linearly polarized light. In the clean $\mathrm{Cu}(332)$ column (b) the ordinate is divided by a factor of 2 with respect to (a) and (c).

$$
P_{Z}=\frac{I_{\uparrow}-I_{\downarrow}}{I_{\uparrow}+I_{\downarrow}} S_{\text {eff }}^{-1}
$$

where $I_{\uparrow, \downarrow}$ denotes the count rates in the two detectors and $S_{\text {eff }}$ is the effective Sherman function. For the present Mott detector $S_{\text {eff }}$ has been calibrated to $S_{\text {eff }}=-0.18{ }^{8}$ The motivation for using the $\mathrm{Cu}(332)$ surface as substrate was the previously determined uniazimuthal alignment of the $(M)-[7] \mathrm{H}$ enantiomer on this stepped surface and potential consequences for the CISS effect. ${ }^{23}$ The center panel in Figure 2 shows results for the clean $\mathrm{Cu}(332)$ surface, the left and right panels for $(M)$ $[7] \mathrm{H}$ and $(P)-[7] \mathrm{H}$, respectively. As expected for a light, nonmagnetic metal, the spin polarization of electrons emitted from the clean copper surface is essentially zero within the error bars for all light polarizations. The [7] H-covered surface shows a distinct enantiospecific finite spin orientation of the electrons, independent of the light polarization employed (Table 1). For excitation with linearly polarized light, which initially generates electrons with both spin orientations at equal amount, the transmission through $(M)-[7] \mathrm{H}$ results in a spin polarization of $P_{Z}=-(6.7 \pm 0.6 \%)$ and through $(P)-[7] \mathrm{H}$ in a spin polarization of $P_{Z}=+(5.5 \pm 0.6 \%)$. For $(M)-[7] \mathrm{H} / \mathrm{Cu}(332)$, excitation with clockwise $(\mathrm{cw})$ and counterclockwise $(\mathrm{ccw})$ circularly polarized light (CPL) results in spin polarizations of $P_{Z}=-(6.0 \pm 0.6 \%)$ and $P_{Z}=-(6.3 \pm 0.6 \%)$, respectively. For the $(P)$-[7] $\mathrm{H}$ layer the polarizations amount to $P_{Z}=+(6.1 \pm$ $0.6 \%)$ and $P_{Z}=+(4.4 \pm 0.6 \%)$ for $\mathrm{cw}$ and $\mathrm{ccw}$ polarization, respectively. In general, this means that electrons with negative spin polarization, that is, those with their spin orientation antiparallel to their momentum, are preferentially transmitted through the $(M)-[7] \mathrm{H}$ monolayer, while electrons transmitted through $(P)-[7] \mathrm{H}$ show an excess of positive spin polarization. Consequently, the preferential direction of the spin polarization of the electrons transmitted through the helicene molecules depends on the enantiomer adsorbed and therefore on the sense of the helicity of the molecules in the monolayer.

The average longitudinal spin polarization of electrons traversing enantiopure $[7] \mathrm{H}$ adsorbed on $\mathrm{Ag}(110)$ is quite similar to those of the copper substrate (Table 1, Supporting Information (SI) Figure S1). With respect to copper, the heavier mass of the silver atoms results in a larger intrinsic spin-orbit coupling (SOC) for the clean surface of -2.5 and $+2.5 \%$ when excited with $\mathrm{cw}$ and ccw CPL, respectively. When using CPL (Table 1) this intrinsic SOC effect adds to the CISS effect induced by the [7] $\mathrm{H}$ monolayers. In sign and magnitude, however, the CISS effect on $\operatorname{Ag}(110)$ in connection with the sense of helicity is basically identical to $\mathrm{Cu}(332)$. The CISS effect was also evaluated in enantiopure helicene monolayers adsorbed on $\mathrm{Au}(111)$. Because of the large SOC of gold the clean substrate shows a significant spin polarization of -24.2 and $+26.7 \%$ when excited with $\mathrm{cw}$ and $\mathrm{ccw}$ CPL, respectively (SI Figure S2). Again, the CISS effect induced by the $[7] \mathrm{H}$ monolayer readily adds to this intrinsic spin polarization: A spin polarization of $+7.9 \%$ results for linearly polarized light for $(P)$ $[7] \mathrm{H} / \mathrm{Au}(111)$ and $-16.0 \%$ and $+34.1 \%$ for $\mathrm{cw}$ and $\mathrm{ccw} \mathrm{CPL}$, respectively (Table 1, SI Figure S2). The $(P)-[7] \mathrm{H}$ increases in all cases the spin polarization of transmitted electrons by $\sim 8 \%$ toward more positive spin, while $(M)-[7] \mathrm{H}$ decreases it toward more negative spin by about the same absolute amount. In particular, a substrate-mediated SOC, which has been suggested to play an important role in CISS ${ }^{28}$ therefore seems to be of no major importance. The total difference in spin polarization induced by the $(M)$ - and $(P)$-enantiomers, $\Delta P_{Z}$, is more or less 
Table 1. Spin Polarization $P_{Z}$ of Transmitted Electrons through Monolayers of [7]H Self-Assembled on Cu(332), $\mathrm{Ag}(110)$, and $\operatorname{Au}(111)$

\begin{tabular}{|c|c|c|c|c|c|c|c|c|c|}
\hline \multirow[b]{2}{*}{ substrate } & \multicolumn{3}{|c|}{$\mathrm{Cu}(332)$} & \multicolumn{3}{|c|}{$\operatorname{Ag}(110)$} & \multicolumn{3}{|c|}{$\mathrm{Au}(111)$} \\
\hline & $M$ & $P$ & $\Delta P_{Z}^{a}$ & $\bar{M}$ & $P$ & $\Delta P_{Z}^{a}$ & $M$ & $\bar{P}$ & $\Delta P_{Z}^{a}$ \\
\hline $\mathrm{cw}$ & -6.0 & +6.1 & 12.1 & -11.9 & +6.3 & 18.2 & -34.8 & -16.0 & 18.8 \\
\hline linear & -6.7 & +5.5 & 12.2 & -9.0 & +7.1 & 16.1 & -8.0 & +7.9 & 15.9 \\
\hline $\mathrm{ccw}$ & -6.3 & +4.4 & 10.7 & -7.1 & +6.2 & 13.3 & +21.8 & +34.1 & 12.3 \\
\hline average $\Delta P_{Z}$ & & & 11.7 & & & 15.9 & & & 15.7 \\
\hline
\end{tabular}

${ }^{a}$ Values are given in $\% ; \Delta P_{Z}=P_{Z}(P)-P_{Z}(M)$

similar for all substrates and amounts to $11.7,15.9$, and $15.7 \%$ for $\mathrm{Cu}(332), \operatorname{Ag}(110)$, and $\mathrm{Au}(111)$, respectively.

In early multiple scattering calculations by Blum and coworkers, randomly oriented gas-phase chiral molecules, such as $\mathrm{H}_{2} \mathrm{~S}_{2}$ and halogenated methane, displayed a finite polarization for scattering of unpolarized electrons. ${ }^{29}$ Similarly, an intensity attenuation of longitudinally polarized electrons was theoretically found when these electrons passed through optically active molecules. ${ }^{30}$ At low electron energies, a decrease of up to $\Delta I \approx 7 \times 10^{-3}$ was calculated, which diminished to about $\Delta I \approx 5 \times 10^{-5}$ at $E=10 \mathrm{eV} .{ }^{30}$ Moreover, it was predicted that scattering on oriented chiral molecules should lead to a stronger increase in the spin polarization as well as a strong attenuation of electrons that are unfavorably longitudinally polarized. Similar multiple scattering calculations have recently been extended to organized self-assembled monolayers by Mujica and coworkers, ${ }^{15}$ where spin polarizations up to $P_{Z}=20 \%$ at certain kinetic energies were predicted for helical model systems.

It seems unlikely that light carbon atoms, which are very little parity-disturbed, are responsible for the observed CISS. It is more probable that extended molecular states with their helical shape enforce electrons onto a helical pathway. To shine light into this latter issue, spin-resolved density functional theory (DFT) calculations including spin-orbit coupling effects have been carried out. ${ }^{31}$ However, a complete description of the CISS effect, which accounts for spin-dependent scattering processes within a standard first-principle approach, is currently not feasible. Hence the theoretical studies were divided here into two parts: (i) identification of the favored adsorption sites, their electronic structure, as well as the strength of spin-orbit coupling in [7] $\mathrm{H}$ by using spin-resolved DFT calculations ${ }^{31}$ and (ii) providing an estimate of the spin polarization by using a previously proposed phenomenological model. ${ }^{13,14}$ Because the latter is now guided by DFT calculations, it is put on a more solid ground than previous attempts. ${ }^{13-15}$

Figure $3 \mathrm{a}$ shows the adsorption configuration and the binding energy of [7] $\mathrm{H}$ on all three substrates. As recently calculated for pentahelicene on $\mathrm{Cu}(111),[7] \mathrm{H}$ binds to the surfaces such that the three proximal $\mathrm{C}_{6}$ rings are oriented parallel to the surface. ${ }^{32}$ This binding geometry has also been found experimentally by X-ray photoelectron diffraction for enantiopure [7] $\mathrm{H}$ on $\mathrm{Cu}(111)$ and $\mathrm{Cu}(332){ }^{23}$ The binding energy $E_{\mathrm{BE}}$ is computed as the difference between the total energy of the helicene plus substrate system $\left(E_{\mathrm{Sub}+\mathrm{Hel}}\right)$ and the individual total energies of the isolated substrate $\left(E_{\mathrm{Sub}}\right)$ and helicene $\left(E_{\mathrm{Hel}}\right)$ molecule: $E_{\mathrm{BE}}=E_{\mathrm{sub}+\mathrm{Hel}}-E_{\mathrm{Sub}}-E_{\mathrm{Hel}}$. A binding energy of $E_{\mathrm{BE}}=-2.22 \mathrm{eV}$ on $\mathrm{Cu}(332)$ indicates a much stronger bond than on $\mathrm{Ag}(110)$ and $\mathrm{Au}(111)$, where [7] $\mathrm{H}$ binds with $E_{\mathrm{BE}}=-1.43$ and $-1.46 \mathrm{eV}$, respectively. The calculated binding energy of helicene on copper compares well with a previous thermal desorption experiment for $[7] \mathrm{H}$ on

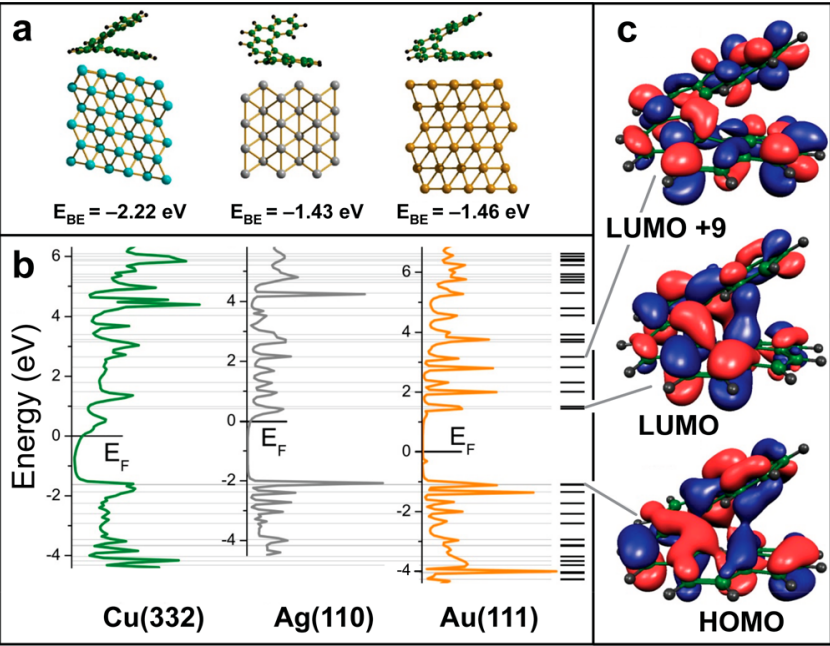

Figure 3. (a) Adsorption configuration and binding energies for [7] helicene deposited on $\mathrm{Cu}(332), \mathrm{Ag}(110)$, and $\mathrm{Au}(111)$. (b) Projected electronic density-of-states and energetic positions of molecular orbitals. (c) Orbital representation of selected electronic states of [7]helicene.

$\mathrm{Cu}(111)$, from which a binding energy of about $E_{\mathrm{BE}}=-2 \mathrm{eV}$ was estimated. $^{33}$

The density of states (DOS) within an energy interval ranging from -4.5 to $+6.0 \mathrm{eV}$ with respect to the Fermi energy $\left(E_{\mathrm{F}}\right)$ is shown in Figure $3 \mathrm{~b}$. The Fermi energy was computed self-consistently for the simulation supercell for each adsorbate geometry (helicene plus substrate). The energetic positions of the occupied and unoccupied molecular states of the isolated helicene are highlighted by horizontal bars on the right-hand side of Figure $3 \mathrm{~b}$. Because of the strong binding energy to copper and partial hybridization of states, the electronic spectrum of helicene deviates significantly from the free molecule. The different relative positions of the Fermi energy are caused by different hybridization of the molecular states with the metallic substrate states and weak charge-transfer effects from the substrate to the molecule, resulting in the Fermi level lying closer to the LUMO level for copper and silver. With an experimentally determined work function for $[7] \mathrm{H} / \mathrm{Cu}(332)$ of $\Phi \approx 4.65 \mathrm{eV}$, it is evident that the UV radiation employed $(h \nu=5.83 \mathrm{eV})$ does not emit electrons out of occupied [7] H states; see the SI and SI Figure S3.

Figure $3 \mathrm{c}$ shows the charge density distribution of selected molecular orbitals of [7] H: HOMO, LUMO, and LUMO+9. While all states show electron densities along the helical backbone of the molecule, the HOMO and LUMO states, for instance, also possess a charge density distribution that directly connects the lower and upper forks of the molecule. Within a simple semiclassical picture, different orbital symmetries will 
have a non-negligible impact on the size of the spin polarization, for example, through the influence of interference effects in the case of states with a nonvanishing amplitude along the helical axis.

In the present experiments, where the photoelectrons travel above the vacuum level, the spatial structure of the high-lying states is especially crucial. There, the electrons are expected to take a helical pathway when propagating through the molecule. One should also notice that a different molecule-substrate electronic coupling leads to different alignment of the molecular orbitals with respect to the corresponding Fermi energy. Hence, depending on the substrate, the molecular orbitals probed in the scattering process for a given photon energy may have different symmetries. Although it is well known that standard DFT approaches are not accurate to describe unoccupied states quantatively, our DFT calculations aim mostly at (i) finding stable conformations of deposited helicene on $\mathrm{Cu}(332), \operatorname{Ag}(110)$, and $\mathrm{Au}(111)$, (ii) determining the level alignment of the molecular orbitals with respect to the substrate Fermi level as well as disclosing the symmetries of relevant molecular orbitals, and (iii) estimating the contribution of spin-orbit interactions in the molecule. In this sense, the precise energetic position of the unoccupied states is not fundamental for the current discussion because they display similar spatial charge density distributions, which are not expected to become strongly modified by more advanced calculations of unoccupied states.

The influence of spin-orbit coupling (SOC) was analyzed in the framework of the Siesta code. ${ }^{3,35}$ Two contributions to the SOC were considered (see the SI and ref 31 for more details): a core-electron related term via pseudopotential, and a term arising from contributions of valence electrons, including the Hartree and the exchange-correlation potentials. While the former contribution is closer to the standard atomic SOC, the second involves the global molecular potential. To simplify the calculations, we considered an idealized 1D helicene "crystal" (an infinite helicene helix with an infinite number of turns) and obtained a spin-orbit splitting of up to $0.75 \mathrm{meV}$ for the top valence band and $0.30 \mathrm{meV}$ for the bottom conduction band. Considering the fact that helicenes comprise a hydrocarbononly system without any chiral centers, these rather large values should be related to the helical character of the system.

Inspired by the DFT studies, the spin polarization of electrons transmitted through the helical system was calculated via a general model Hamiltonian. ${ }^{14}$ The model uses a coarsegrained representation of the helicene molecule, assuming within a quasi-classical picture that electrons are probing a helical pathway and that the overall molecular electrostatic potential $U(r)$ induces a helical electric field $E(r)=-\nabla U(r)$ responsible for the SOC. For the helical scattering pathway, a radius $R_{0}=3 \AA$ and a pitch of $b=3.5 \AA$ was considered (see the SI and ref 14 for additional technical details and the formulation of the model).

Results for the spin polarization with an unpolarized incoming state for various numbers of helical turns $L$ are shown in Figure 4. The spin polarization displays, in general, relatively large absolute values reaching up to $P_{Z}=10-15 \%$; its sign, however, strongly varies as a function of the incoming electron energy. Similar qualitative results (not shown) were obtained for the case that in addition a transport pathway emerges along the helical axis. The peak structure of the spin polarization is closely related to the coherent nature of the electronic and spin transport and also reflects the presence of

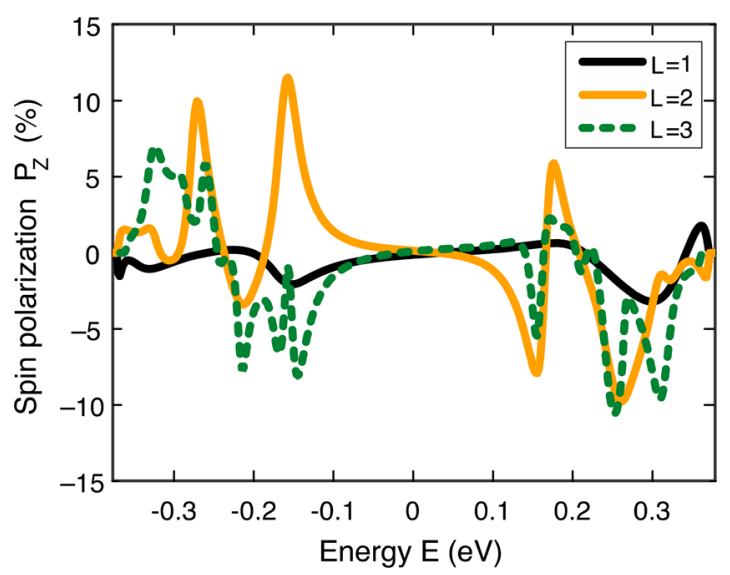

Figure 4. Length and energy dependence of spin polarization for the model helical system computed for different numbers of left-handed helical turns $\mathbf{L}$. The strong energy dependence reflects the discrete structure of the electronic spectrum of the tight-binding model.

molecular resonances. Its energy sensitivity is expected to be much less pronounced in the case that dephasing becomes inportant because the interaction with a dissipative environment will strongly broaden the electronic states. In which case a larger spin polarization may result will strongly depend on the individual coupling parameters. Different numbers of turns (from $L=1$ to $L=2$ and 3 ) increase the spin polarization; there is, however, no clear energy-independent trend. For states below $E=0$ (corresponding to the Fermi energy of the system) the sign can vary for different $L$. Above $E=0$ the tendency is smoother and the spin polarization has mostly negative values (note that only the relative position of the states with respect to the Fermi level is relevant). The absolute bandwidths in these calculations are much less relevant because they depend on the choice of the nearest-neighbor coupling in the used tightbinding model.

For a single left-handed helical turn at $E=+0.3 \mathrm{eV}$ above the Fermi level a longitudinal spin polarization of $P_{Z} \approx-3 \%$ and $P_{Z}$ $\approx+0.1 \%$ (not shown) is obtained for the pure helical as well as for the combined helical/straight electron paths, respectively. The size and sign of the polarization for the helical path is, despite the simplicity of the model, in reasonable agreement with the experimental results. The change in polarization when including an additional coupling along the helical axis may be related to quantum interference effects. Because in the coherent regime the polarization at each energy is sensitively depending on the transmission for spin-up and spin-down states, quantum interference effects can be expected to affect the polarization. In the present case, we found a sensitive suppression of the polarization. The role of interference effects requires, however, a separate study, also with energy-resolved experimental spin polarization data. Increasing the number of molecular turns increases the spin polarization up to $\left|P_{Z}\right| \approx 10 \%$. This clearly points to the importance of the helical field acting on the electrons during the transport process through the molecule to obtain efficient spin filtering. In these calculations a change of the helicity in both the quasi-classical electron path and the helical electric field causes a change of sign of the spin polarization.

A similar calculation was recently carried out within a different model by Sun and coworkers for eicosahelicene, that is, $20[\mathrm{a}, \mathrm{c}]$-annulated benzo rings with three full helical turns. ${ }^{20}$ Using Büttiker probes for describing dephasing effects, a 
significant spin-orbit splitting of $5.1 \mathrm{meV}$, and a relatively large dephasing strength of $27 \mathrm{meV}$, they mimicked hopping-like motion through this aromatic $\pi$-conjugated molecule. In their model, dephasing effects were required to achieve any spin filtering. ${ }^{16}$ Longitudinal spin polarizations up to $P_{Z}=25 \%$ were obtained for electron energies between $E=0.5$ and $1.0 \mathrm{eV}$ below and above the Fermi energy. Importantly, Sun et al. found opposite spin polarizations for the enantiomers at same electron energies. However, their spin polarization changes sign over narrow intervals of $\sim 100 \mathrm{meV}$.

In conclusion, we have shown experimentally for the first time that the direction of preferentially transmitted longitudinal electron spin depends on the sign of the helicity of a chiral molecule. Density-functional-based calculations provided an order of magnitude estimate of the spin-orbit coupling strength in helicene, supporting previous values used in model Hamiltonian calculations. ${ }^{13,14}$ Subsequent model calculations, assuming that spin-orbit interaction induced by a helical electric field is the major source of spin polarization, agree qualitatively with the observed experimental results in terms of the connection of the polarization to the molecular helicity as well as the spin direction of electrons and the magnitude of the polarization. Beyond the previously studied molecules, such as DNA, bacteriorhodopsin, and oligo-peptides with rather low hopping-type conduction, it is shown here that transmission through a molecular monolayer of an aromatic helical molecule with high conductivity leads to substantially spin-polarized electrons. The fact that the values of the spin polarization are very similar for the three substrates with very different spin-orbit coupling strengths provides a strong demonstration that the dominant factor for spin filtering is the chiral system and not, for example, spin-orbit coupling effects with the substrate as previously suggested.

\section{EXPERIMENTAL METHODS}

Experimental details can be found in the Supporting Information. For spin measurements the fifth harmonic of a neodymium laser at $\lambda=213 \mathrm{~nm}(h \nu=5.83 \mathrm{eV})$ and for ultraviolet photoemission its ninth harmonic at $\lambda=118.2 \mathrm{~nm}$ $(h \nu=10.5 \mathrm{eV})$ were employed. The samples were prepared and measured in ultrahigh vacuum with a residual pressure of $10^{-9}$ mbar. The photoelectrons from the sample with energies up to $1.15 \mathrm{eV}$ were collectively drawn into a $90^{\circ}$ bender. The electron spin state was analyzed with a Mott polarimeter, accelerating the electrons to $50 \mathrm{keV}^{7}$ For the histograms in Figure 2, we recorded 120 spin polarizations from $(M)-17 \mid \mathrm{H} / \mathrm{Cu}(332)$ and 76 spin polarizations from $(P)-17 \mid \mathrm{H} / \mathrm{Cu}(332)$. The given experimental accuracy of the spin polarization is obtained from the standard deviation of the mean of the spin polarization. [7] $\mathrm{H}$ was sublimated at $160{ }^{\circ} \mathrm{C}$ for up to 35 min from an evaporation cell (Kentax, TCE-BSC) and deposited onto the respective single crystal, which was kept at a temperature of $200{ }^{\circ} \mathrm{C}$. Heating above $125^{\circ} \mathrm{C}$ leads to the desorption of all multilayers of [7] $\mathrm{H}^{33}$ An aperture in front of the single crystal prevented condensation of [7] $\mathrm{H}$ onto the molybdenum reference sample.

\section{ASSOCIATED CONTENT}

\section{S Supporting Information}

The Supporting Information is available free of charge on the ACS Publications website at DOI: 10.1021/acs.jpclett.8b00208.
Spin polarization measurements of monolayers of $(M)$ [7] $\mathrm{H}$ and $(P)-[7] \mathrm{H}$ on $\mathrm{Ag}(110)$ and on $\mathrm{Au}(111)$ as well as on the clean $\operatorname{Ag}(110)$ and $\mathrm{Au}(111)$ surfaces. UPS on $[7] \mathrm{H} / \mathrm{Cu}(332)$. Detailed description of the experimental setup and the theoretical modeling approaches. (PDF)

\section{AUTHOR INFORMATION}

\section{Corresponding Authors}

*K.-H.E.: E-mail: karl-heinz.ernst@empa.ch.

*H.Z.: E-mail: hzach@uni-muenster.de.

ORCID

Gianaurelio Cuniberti: 0000-0002-6574-7848

Karl-Heinz Ernst: 0000-0002-2077-4922

\section{Notes}

The authors declare no competing financial interest.

\section{ACKNOWLEDGMENTS}

G.C. and H.Z. acknowledge financial support from the Volkswagen Stiftung (grant nos. 88366 and 88364, respectively). K.-H.E. and J.S. thank the Swiss National Science Foundation (SNSF) for support (grant nos. 200020_144339 and 200020_163296). This work was partly supported by the German Research Foundation (DFG) within the Cluster of Excellence "Center for Advancing Electronics Dresden". We acknowledge the Center for Information Services and High Performance Computing ( $\mathrm{ZIH})$ at TU Dresden for computational resources.

\section{REFERENCES}

(1) Siegel, J. S. Homochiral Imperative of Molecular Evolution. Chirality 1998, 10, 24-27.

(2) Blaser, H.-U. Enantioselective Synthesis Using Chiral Heterogeneous Catalysts. Tetrahedron: Asymmetry 1991, 2, 843-866.

(3) Lemieux, R. P. Chirality Transfer in Ferroelectric Liquid Crystals. Acc. Chem. Res. 2001, 34, 845-853.

(4) Dreiling, J. M.; Lewis, F. W.; Mills, J. D.; Gay, T. J. Anomalously Large Chiral Sensitivity in the Dissociative Electron Attachment of 10Iodocamphor. Phys. Rev. Lett. 2016, 116, 093201.

(5) Campbell, D. M.; Farago, P. S. Spin-Dependent Electron Scattering from Optically Active Molecules. Nature 1985, 318, 52-53.

(6) Mayer, S.; Kessler, J. Experimental Verification of Electron Optic Dichroism. Phys. Rev. Lett. 1995, 74, 4803-4806.

(7) Göhler, B.; Hamelbeck, V.; Markus, T.; Kettner, M.; Hanne, G.; Vager, Z.; Naaman, R.; Zacharias, H. Spin Selectivity in Electron Transmission Through Self-Assembled Monolayers of DoubleStranded DNA. Science 2011, 331, 894-897.

(8) Kettner, M.; Göhler, B.; Zacharias, H.; Mishra, D.; Kiran, V.; Naaman, R.; Fontanesi, C.; Waldeck, D. H.; Sęk, S.; Pawlowski, J.; et al. Spin Filtering in Electron Transport Through Chiral Oligopeptides. J. Phys. Chem. C 2015, 119, 14542-14547.

(9) Wolf, S. A.; Awschalom, D. D.; Buhrman, R. A.; Daughton, J. M.; von Molnár, S.; Roukes, M. L.; Chtchelkanova, A. Y.; Treger, D. M. Spintronics: A Spin-Based Electronics Vision for the Future. Science 2001, 294, 1488-1495.

(10) Rosenberg, R. A.; Mishra, D.; Naaman, R. Chiral Selective Chemistry Induced by Natural Selection of Spin-Polarized Electrons. Angew. Chem. 2015, 127, 7403-7406.

(11) Ray, K.; Ananthavel, S. P.; Waldeck, D. H.; Naaman, R. Asymmetric Scattering of Polarized Electrons by Organized Organic Films of Chiral Molecules. Science 1999, 283, 814-816.

(12) Yeganeh, S.; Ratner, M. A.; Medina, E.; Mujica, V. Chiral Electron Transport: Scattering Through Helical Potentials. J. Chem. Phys. 2009, 131, 014707. 
(13) Gutierrez, R.; Díaz, E.; Naaman, R.; Cuniberti, G. Spin-Selective Transport Through Helical Molecular Systems. Phys. Rev. B: Condens. Matter Mater. Phys. 2012, 85, 081404.

(14) Gutierrez, R.; Diaz, E.; Gaul, C.; Brumme, T.; DominguezAdame, F.; Cuniberti, G. Modeling Spin Transport in Helical Fields: Derivation of an Effective Low-Dimensional Hamiltonian. J. Phys. Chem. C 2013, 117, 22276-22284.

(15) Medina, E.; Lopez, F.; Ratner, M. A.; Mujica, V. Chiral Molecular Films as Electron Polarizers and Polarization Modulators. Europhys. Lett. 2012, 99, 17006.

(16) Guo, A.-M.; Sun, Q.-F. Spin-Selective Transport of Electrons in DNA Double Helix. Phys. Rev. Lett. 2012, 108, 218102.

(17) Matityahu, S.; Utsumi, Y.; Aharony, A.; Entin-Wohlman, O.; Balseiro, C. A. Spin- Dependent Transport Through a Chiral Molecule in the Presence of Spin-Orbit Interaction and Nonunitary Effects. Phys. Rev. B: Condens. Matter Mater. Phys. 2016, 93, 075407.

(18) Yang, Y.; da Costa, R. C.; Fuchter, M. J.; Campbell, A. J. Circularly Polarized Light Detection by a Chiral Organic Semiconductor Transistor. Nat. Photonics 2013, 7, 634-638.

(19) Kiran, V.; Mathew, S. P.; Cohen, S. R.; Hernández Delgado, I.; Lacour, J.; Naaman, R. Helicenes - A New Class of Organic Spin Filter. Adv. Mater. 2016, 28, 1957-1962.

(20) Pan, T.-R.; Guo, A.-M.; Sun, Q.-F. Spin-Polarized Electron Transport Through Helicene Molecular Junctions. Phys. Rev. B: Condens. Matter Mater. Phys. 2016, 94, 235448.

(21) Shen, Y.; Chen, C.-F. Helicenes: Synthesis and Applications. Chem. Rev. 2012, 112, 1463-1535.

(22) Ernst, K.-H. Stereochemical Recognition of Helicenes on Metal Surfaces. Acc. Chem. Res. 2016, 49, 1182-1190.

(23) Fasel, R.; Cossy, A.; Ernst, K.-H.; Baumberger, F.; Greber, T.; Osterwalder, J. Orientation of Chiral Heptahelicene $\mathrm{C}_{30} \mathrm{H}_{18}$ On Copper Surfaces: An X-Ray Photoelectron Diffraction Study. J. Chem. Phys. 2001, 115, 1020-1027.

(24) Fasel, R.; Parschau, M.; Ernst, K.-H. Chirality Transfer From Single Molecules Into Self-Assembled Monolayers. Angew. Chem., Int. Ed. 2003, 42, 5178-5181.

(25) Seibel, J.; Zoppi, L.; Ernst, K.-H. 2D Conglomerate Crystallization of Heptahelicene. Chem. Commun. (Cambridge, U. K.) 2014, 50, 8751-8753.

(26) Seibel, J.; Parschau, M.; Ernst, K.-H. From Homochiral Clusters to Racemate Crystals: Viable Nuclei in 2D Chiral Crystallization. J. Am. Chem. Soc. 2015, 137, 7970-7973.

(27) Seibel, J.; Parschau, M.; Ernst, K.-H. Two-Dimensional Crystallization of Enantiopure and Racemic Heptahelicene on $\mathrm{Ag}(111)$ and $\mathrm{Au}(111)$. J. Phys. Chem. C 2014, 118, 29135-29141.

(28) Gersten, J.; Kaasbjerg, K.; Nitzan, A. Induced Spin Filtering in Electron Transmission Through Chiral Molecular Layers Adsorbed on Metals with Strong Spin-Orbit Coupling. J. Chem. Phys. 2013, 139, 114111.

(29) Busalla, A.; Blum, K.; Thompson, D. G. Differential Cross Section for Collisions Between Electrons and Oriented Chiral Molecules. Phys. Rev. Lett. 1999, 83, 1562-1565.

(30) Fandreyer, R.; Thompson, D.; Blum, K. Attenuation of Longitudinally Polarized Electron Beams by Chiral Molecules. J. Phys. B: At., Mol. Opt. Phys. 1990, 23, 3031-3040.

(31) Maslyuk, V. V.; Gutierrez, R; Cuniberti, G. Spin-Orbit Coupling in Nearly Metallic Chiral Carbon Nanotubes: A Density-Functional Based Study. Phys. Chem. Chem. Phys. 2017, 19, 8848-8853.

(32) Mairena, A.; Zoppi, L.; Seibel, J.; Tröster, A. F.; Grenader, K.; Parschau, M.; Terfort, A.; Ernst, K.-H. Heterochiral to Homochiral Transition in Pentahelicene 2D Crystallization Induced by SecondLayer Nucleation. ACS Nano 2017, 11, 865-871.

(33) Ernst, K.-H.; Kuster, Y.; Fasel, R.; Müller, M.; Ellerbeck, U. Two-Dimensional Separation of [7]Helicene Enantiomers on $\mathrm{Cu}(111)$. Chirality 2001, 13, 675-678.

(34) Soler, J.; Artacho, E.; Gale, J.; García, A.; Junquera, J.; Ordejón, P.; Sánchez-Portal, D. The SIESTA Method for $a b$ initio Order-N Materials Simulation. J. Phys.: Condens. Matter 2002, 14, 2745-2779.
(35) Fernández-Seivane, L.; Oliveira, M. A.; Sanvito, S.; Ferrer, J. OnSite Approximation for Spin-Orbit Coupling in Linear Combination of Atomic Orbitals Density Functional Methods. J. Phys.: Condens. Matter 2006, 18, 7999-8013. 